\title{
Molecular markers associated with response to chemotherapy in gastro- entero-pancreatic neuroendocrine tumors
}

\author{
Dermot O'Toole ${ }^{1,2 \star}$, Anne Couvelard ${ }^{3,4 *}$, Vinciane Rebours ${ }^{2}$, \\ Magali Zappa ${ }^{5}$, Olivia Hentic ${ }^{2}$, Pascal Hammel ${ }^{2}$, Philippe Levy ${ }^{2}$, \\ Pierre Bedossa ${ }^{3,4}$, Eric Raymond ${ }^{6}$ and Philippe Ruszniewski ${ }^{2}$
}

\author{
${ }^{1}$ Department of Clinical Medicine and Gastroenterology, St James's Hospital and Trinity College Dublin, James's Street, \\ Dublin 8, Ireland \\ ${ }^{2}$ Service de Gastroentérologie, Pôle des Maladies de l'Appareil Digestif and ${ }^{3}$ Service d'Anatomie Pathologique, AP-HP, Hôpital \\ Beaujon, Clichy Cedex 92110, France \\ ${ }^{4}$ INSERM U773, Centre de Recherche Biomédicale Bichat-Beaujon, CRB3, Paris F-75018, France \\ ${ }^{5}$ Service de Radiologie and ${ }^{6}$ Service d'Oncologie Bichat-Beaujon, Hôpital Beaujon, Clichy Cedex 92110, France
}

(Correspondence should be addressed to D O'Toole at Department of Clinical Medicine and Gastroenterology, St James's Hospital and Trinity College; Email: dermot.otoole@tcd.ie)

*(D O'Toole and A Couvelard contributed equally to this work)

\begin{abstract}
Response rates to cytotoxics in gastro-entero-pancreatic neuroendocrine tumors (GEP-NETs) vary; recent trials demonstrated lack of objective response rates in up to $70 \%$ of patients. Identification of predictive therapeutic biomarkers would be beneficial in the treatment of GEP. Selected markers with known or suspected capability of predicting response to cytotoxics or prognosis (Ki-67, p53, multidrug resistance protein-1 (MDR1), Akt, thymidylate synthase (TS), phosphatase and tensin homolog (PTEN), CA9, cluster of differentiation 34 (CD34), vascular endothelial growth factor (VEGF), hypoxia-inducible factor (HIF)-1, mismatch repair gene - human mutL homolog 1 (hLMH1), and $\mathrm{Bcl}-2$ ) were analyzed using immunohistochemisrtry in 60 treatment-naive patients receiving chemotherapy $(n=46)$ or chemoembolization $(n=14)$ for inoperable advanced and/or metastatic GEP and correlated with prognosis (survival and response rates). Therapy included systemic chemotherapy with streptozotocin $(n=28)$, doxorubicin $(n=14), 5$-fluorouracil $(n=18)$, and etoposide/cisplatinum ( $n=16$ ), or chemoembolization (streptozotocin, 9; doxorubicin, 5). Factors associated with overall survival in the entire cohort were Ki-67, $P<0.001$; tumor grade, $P<0.001$; tumor differentiation, $P<0.001$; CA9, $P=0.004$; Akt, $P=0.01$; HIF-1, $P<0.001$; p53, $P<0.0001$; and $\mathrm{hMLH} 1, P=0.005$. Markers associated with treatment response included overall group: Akt and PTEN ( $P=0.05$ and 0.05 respectively); streptozotocin: Akt $(P=0.07)$, TS $(P=0.02)$, and PTEN $(P=0.017)$; doxorubicin: Ki-67 $(P=0.05)$, Akt $(P=0.06)$, and CA9 $(P=0.02)$. At multivariate analysis, Akt was significantly associated with a nonresponse to therapy (objective response (OR): $0.2(0.05-0.8)$ ). For patients receiving only systemic chemotherapy $(n=46)$, PTEN (0.04) and hLMH1 (0.03) were correlated with treatment response and for individual molecules were streptozotocin: PTEN $(P=0.008)$ and hLMH1 (0.07); doxorubicin: Akt $(P=0.09)$, CA9 $(P=0.09)$, and hLMH1 $(P=0.09)$. These results demonstrate a number of new prognostic biomarkers in GEP-NET, and in addition, response to chemotherapy was correlated with a simple panel of selected markers (such as CA9, Akt, PTEN, TS, and hLMH1).
\end{abstract}

Endocrine-Related Cancer (2010) 17 847-856

\section{Introduction}

Advances in treating solid tumors include molecular targeting agents and improved cytotoxic combinations. Nonetheless, most cytotoxics have short-lived effects, and inherent and/or acquired resistance gradually develops. Individualized therapy according to a patient's predictive markers analysis should optimize tailoring certain drugs. This approach is currently 
gaining popularity in patients with colorectal cancer (CRC) where KRAS mutations confer lack of benefit to monoclonal therapy against epidermal growth factor (EGF) receptor (Allegra et al. 2009). Again in CRC, the downstream regulator of EGF, PTEN, has been suggested to predict resistance to cetuximab in combination with irinotecan (Loupakis et al. 2009). Therapeutic responses to cytotoxics in patients with gastro-entero-pancreatic neuroendocrine tumors (GEP-NETs) vary with best responses attributable to streptozotocin-based regimens especially in pancreatic GEP (O'Toole et al. 2004, O'Toole 2006). Nonetheless, recent clinical trials have demonstrated lack of objective response rates in up to $60-70 \%$ of patients even with pancreatic tumors (O'Toole et al. 2004). Identification of markers predictive of tumor response would clearly be beneficial here. The corollary is also true as markers capable of identifying a lack of therapeutic benefit that would prevent the use of potentially harmful cytotoxics allowing patients to be selected for other therapeutics.

Markers such as the Ki-67 and p53 are associated with aggressive GEP-NETs but their influence on therapy is unknown. Several putative molecular markers predicting either sensitivity or resistance to oncological therapeutics have been proposed. The multidrug resistance protein and other ABC transporters have well-documented implications in cancer resistance, and attempts at modulating these drug transporters have met with some success (Modok et al. 2006). Little is known of MDR1 in GEP tumors, although we recently found that MDR1 is associated with an aggressive phenotype in a gene expression profiling study in pancreatic GEP (Couvelard et al. 2006). High thymidylate synthase (TS) protein and mRNA expression have been associated with poor clinical outcome in patients treated with 5-fluorouracil (5-FU)-based protocols in esophageal (Harpole et al. 2001), breast (Pestalozzi et al. 1997), bladder (Mizutani et al. 2001), and non-small cell lung cancers (Huang et al. 2000). The same has been applied to CRC, although with conflicting results (Lurje et al. 2009). Similarly, the protein kinase Akt, an important player in phosphatidylinositol-3 kinase activation (cell proliferation, growth, and survival), has been identified as a possible maker of response and survival in some tumors. pAkt has been associated with disease progression and survival in patients with melanoma (Dhawan et al. 2002). Chemotherapy-induced Akt has been found to confer acquired resistance in many cellular systems (Huang \& Hung 2009). Hypoxiaregulating proteins have also been implicated in poor response to therapy and more aggressive tumor behavior in some cancers. In breast cancer, both the hypoxia-inducible factor (HIF)- $1 \alpha$ and CA9 were associated with aggressive tumors and CA9-positive phenotype with a poor response to therapy in a subset of cancers (Tan et al. 2009). In a non-small cell lung cancer model, chemoresistance to doxorubicin has been partially mediated via enhancement of HIF- $1 \alpha-$ mediated angiogenesis (Mi et al. 2008). We have recently demonstrated that HIF-regulatory proteins are highly expressed in pancreatic GEP, and are correlated with tumor metastases, recurrence, and prognosis (Couvelard et al. 2005, 2008). Mismatch repair deficiency proteins (hLMH1 and heat shock proteins) have been associated with drug resistance to doxorubicin and cisplatin: hLMH1 was correlated with an aggressive phenotype in gastric cancers (Kitajima et al. 2003), and has been associated with resistance to chemotherapy in germ cell tumors (Honecker et al. 2009) and poor response to adjuvant 5-FU in patients with CRC (Ide et al. 2008). Similarly, Bcl-2 has been reported to confer cytotoxic drug resistance in patients with CRC (Bendardaf et al. 2008).

We examine a number of selected markers with known or suspected involvement in predicting response to cytotoxics in a group of patients treated with inoperable advanced and/or metastatic GEP-NET.

\section{Methods}

\section{Patients}

Patients treated at a single institution were included through an institutional review board approved protocol where patients provide informed consent for the use of medical records, biospecimens, and clinical outcome data for medical research purposes. Medical and/or clinical trial records were used to obtain demographic and treatment information as well as to assess response to chemotherapy. Sixty patients whose tissue biopsy specimens and complete follow-up data were available were included (Table 1). All patients had been reviewed at a multidisciplinary tumor board (MTB), and had advanced or metastatic GEP tumors prior to commencing chemotherapy (primary site, Table 1). Tissue samples included either fine core liver biopsies or surgically obtained wedge samples.

\section{Chemotherapy}

The chemotherapy regimen was based on tumor differentiation and site of primary tumor as part of the MTB. Cytotoxics for patients with well-differentiated tumors included streptozotocin with either doxorubicin or 5-FU, $n=32$; or intra-arterial 
Table 1 Patient's demographics and main pathologic features

\begin{tabular}{ll}
\hline Total number of patients & 60 \\
Sex & \\
Female & $34(57 \%)$ \\
Male & $26(43 \%)$ \\
Tumor morphology & \\
Well-differentiated carcinomas & $47(78 \%)$ \\
Poorly differentiated carcinomas & $13(22 \%)$ \\
Tumor grade (grading according to TNM 2006/2007) \\
Grade 1 & $24(40 \%)$ \\
Grade 2 & $23(38 \%)$ \\
Grade 3 & $13(22 \%)$ \\
Tumor origin & \\
Pancreas & $29(48 \%)$ \\
Gastric & $3(5 \%)$ \\
lleum & $12(20 \%)$ \\
Colon & $4(7 \%)$ \\
Rectum & $1(2 \%)$ \\
Hepatic metastases of unknown primary & $11(18 \%)$ \\
Follow-up & \\
Duration, median (months) & 42 \\
Range (months) & $1-155$ \\
Lost to follow-up & 0 \\
Death & $31(52 \%)$ \\
\hline
\end{tabular}

${ }^{\text {aproposed classification }}{ }^{22,23}$.

chemoembolization in 12 patients with liver metastases as the only disease site using either streptozotocin $(n=7)$ or doxorubicin $(n=5)$. Etoposide and cisplatin $(n=16)$ were used for all patients with poorly differentiated tumors and in addition in three with well-differentiated carcinomas due to a high Ki-67 and an aggressive clinical nature (Rindi et al. 2006, 2007). Patients receiving etoposide and cisplatinum always had this doublet, and therefore, analysis of these molecules independently was not possible.

\section{Response to therapy}

Response to therapy was defined following first-line treatment after completion of a first full course (WHO criteria), i.e. four cycles of streptozotocin with either doxorubicin or 5-FU at 6 weekly intervals; four cycles of etoposide and cisplatinum at 4 weekly intervals; and following a single chemoembolization 3 months following therapy. For purpose of analysis in this paper, patients with a complete or partial response were grouped as responders, whereas patients with stabilization or progression were grouped as nonresponders. Patients who progressed during the first chemotherapy course were deemed nonresponders.

\section{Evaluation of tumor marker}

Archival GEP tumor tissue specimens were identified through a review of pathology records. Paraffin sections $(4 \mu \mathrm{m})$ were used for immunohistochemical staining.
Two pathologists reviewed the slides and reached consensus for marking and scoring; pathologists were blinded to all clinical and patient outcomes.

\section{Antibodies and immunohistochemical techniques}

All antibodies are reactive in paraffin-embedded sections (Table 2). Immunostaining of paraffin sections was performed after dewaxing and rehydrating slides. Antigen retrieval was conducted by pretreatment with high temperature. An automatized technique (Streptavidin peroxidase with an automated Ventana Benchmark, Ventana Medical Systems, Tucson, AZ, USA) was used for the detection of all molecules.

\section{Ki-67}

In resected specimens, Ki-67 was calculated in an area of high staining, chosen at a low optical power $(\times 10)$, in three high-power fields $(\times 40)$ containing at least 2000 cells (Rindi et al. 2006, 2007). In biopsy specimens, it was calculated in the entire surface of the specimen. The median Ki-67 was 4.5, and high values were thus considered above $5 \%$.

\section{Microvascular density (MVD)}

Quantification of microvessel density was performed using cluster of differentiation 34 (CD34) antibody. In resection specimens, it was calculated in hot spots, whereas in biopsies it was calculated in the entire fragment (which represented at least two high power fields, objective $\times 40,0.5 \mathrm{~mm}^{2}$ ). In five biopsies, counting was not feasible because the surface was $<0.5 \mathrm{~mm}^{2}$. Vessels with a clearly defined lumen or well-defined linear vessel shape were taken into account. The median value was 100 , and values higher than this were considered as high.

\section{Scoring methods}

For other antibodies tested, immunohistochemistry was evaluated by semiquantitative manner. A score was obtained by multiplying the intensity (negative score as 0 , weak score as 1 , and strong score as 2) by percentage of stained cells (0-100\%). A median score was calculated for each protein, and tumors with a score greater than the median value were considered at high protein expression. For p53, a score $>5$ was considered at high protein expression. Staining of stroma and microvessels was also recorded as negative or positive. 
Table 2 Antibodies used in the study

\begin{tabular}{lllll}
\hline Antibodies & Dilution & $\begin{array}{c}\text { Monoclonal }(\mathrm{M}) \\
\text { or polyclonal }(\mathrm{P})\end{array}$ & Clone & Source/reference number \\
\hline Ki-67 & $1 / 400$ & $\mathrm{M}$ & MIB-1; IgG1 & DAKO/M7240 \\
MDR1 & $1 / 200$ & $\mathrm{M}$ & $\mathrm{G}-1$ & Santa Cruz Biotechnology/sc-13131 \\
Akt2 & $1 / 500$ & $\mathrm{P}$ & & Cell Signalling Technologies/4057 \\
VEGF & $1 / 300$ & $\mathrm{P}$ & & Santa Cruz Biotechnology/sc-152 \\
CD34 & $\mathrm{M}$ & $\mathrm{QBEND} 10$ & Immunotech/786 \\
HIF-1 & $1 / 500$ & $\mathrm{M}$ & 54 & BD Biosciences/610958 \\
CA9 & $1 / 20$ & $\mathrm{P}$ & & Novus Biological/NB100-417 \\
PTEN & $1 / 200$ & $\mathrm{M}$ & $\mathrm{A} 2 \mathrm{~B} 1$ & Santa Cruz Biotechnology/sc-7974 \\
Thymidylate synthase & $1 / 50$ & $\mathrm{M}$ & TS106/4H4B1 & ZYMED/18-0405 \\
p53 & $1 / 25$ & $\mathrm{M}$ & $\mathrm{DO} 7$ & DAKO/M7001 \\
hMLH1 & $1 / 400$ & $\mathrm{M}$ & $\mathrm{G} 168-728$ & BD Pharmingen/554073 \\
Bcl-2 & $1 / 50$ & $\mathrm{M}$ & $124-3$ & DAKO/M887
\end{tabular}

DAKO, Ely, Cambridgeshire, UK; Santa Cruz Biotechnology, Santa Cruz, CA, USA; Cell Signalling Technologies, Beverly, MA, USA; Immunotech, Marseille, France; ZYMED, Carpinteria, CA, USA; BD Pharmingen, San Jose, CA, USA.

\section{Statistical analysis}

Patients' categorical data are presented as median with range. Differences were assessed using the Kruskal-Wallis test for continuous data, and $\chi^{2}$ test or Fisher's exact test for categorical data. A multivariate model as a logistic regression was performed to determine data associated with chemotherapy response. Overall survival was defined as the time from initiation of chemotherapy until death from any cause. The end point of the follow-up period was January 2008. No patients were lost to follow-up. The survival analyses for censored data were performed using the KaplanMeier method for overall survival. The Logrank test was used to compare survival data in relation to individual markers. Data were analyzed with the SAS 9.1 software (SAS Institute Inc., Cary, NC, USA). All statistical tests were two-sided. The critical level of statistical significance was set at a $P$ value $<0.05$.

\section{Results}

\section{Differentiation and grading}

There were 47 well-differentiated and 13 poorly differentiated carcinomas. When the recently proposed tumor, node, metastasis (TNM) classification and grading system (Rindi et al. 2006, 2007) were applied, 24 patients had grade 1, 23 had grade 2, and 13 had grade 3.

\section{Expression of markers}

\section{Ki-67}

Ki-67 ranged from 0 to $90 \%$ (median: 4.5; mean: 14.3; Fig. 1a); $50 \%$ of tumors had high Ki-67. High Ki-67 correlated with poorly differentiated tumors $(P<0.001)$.

\section{Microvessel}

MVD ranged from 0 to 350 vessels $/ \mathrm{mm}^{2}$ (median: 100; mean: 126; Fig. 1b); $42 \%$ had high expression. MVD was higher in tumors of grade $\mathrm{G}_{2} / \mathrm{G}_{3}$ compared with $\mathrm{G}_{1}$ tumors $(P=0.02)$.

\section{p53}

The nuclear score ranged from 0 to 200 (median: 0; mean: 18.8); $24 \%$ had high nuclear expression (Fig. 1c). There was no expression of p53 in cytoplasm, stroma, or vessels. High p53 correlated with poorly differentiated tumors $(P<0.001)$ and grade $\mathrm{G}_{2} / \mathrm{G}_{3}$ tumors $(P<0.001)$.

\section{HIF-1 $\alpha$}

The nuclear score ranged from 0 to 80 (median: 57.5; mean: 11.6); 27\% had high expression (Fig. 1d). There was no expression of HIF- $1 \alpha$ in cytoplasm, stroma, or vessels. Higher HIF-1 $\alpha$ correlated with poorly differentiated $(P<0.001)$ and grade $\mathrm{G}_{2} / \mathrm{G}_{3}$ tumors $(P=0.004)$.

\section{VEGF}

The cytoplasmic score ranged from 0 to 200 (median: 50; mean: 136; Fig. 1e); $45 \%$ had high staining. There was no expression of VEGF in nuclei or stroma. Some vessels were positive but this was ignored. No correlation with tumor grade or differentiation was noted.

\section{Akt}

The cytoplasmic score ranged from 0 to 200 (median: 100; mean: 126; Fig. 1f); 46\% were considered high. There was no expression of Akt in nuclei, stroma, or vessels. Some vessels were positive but this was not taken into account. Higher Akt correlated with poorly differentiated tumours $(P=0.01)$. 

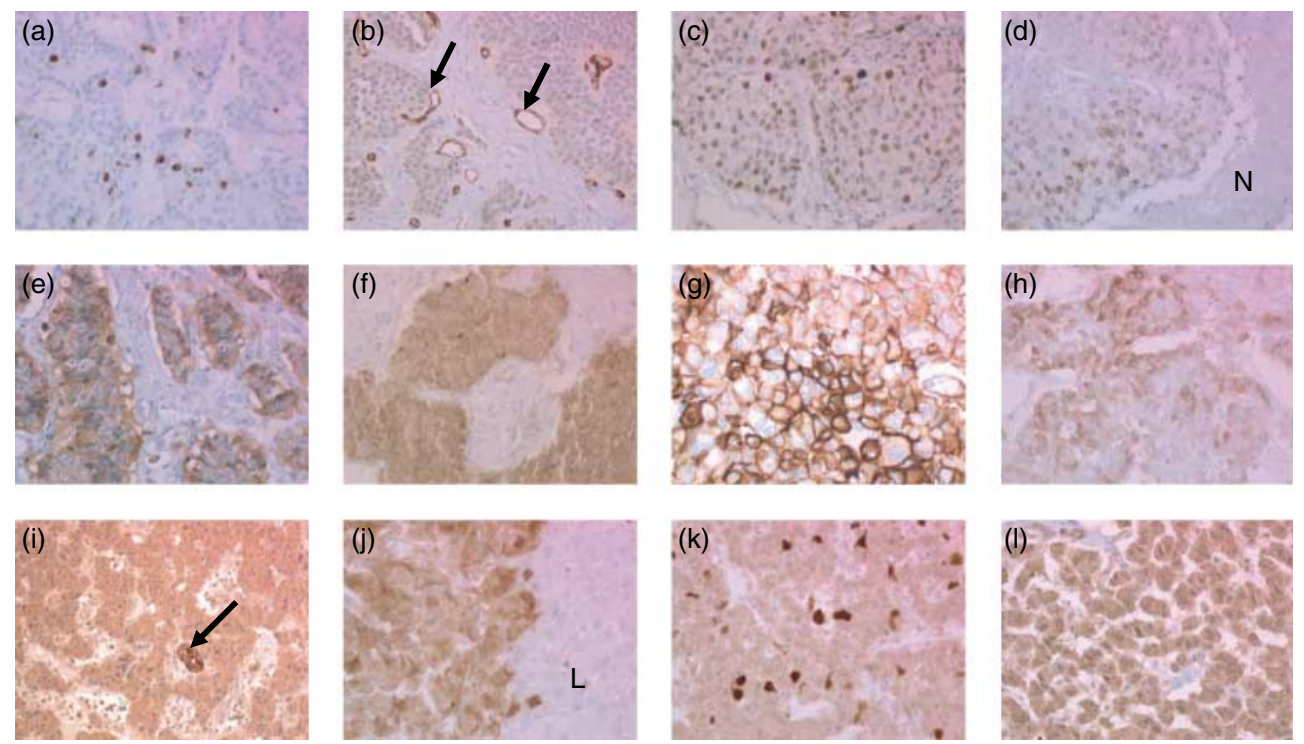

Figure 1 Immunohistochemical expression of the 12 markers in gastro-entero-pancreatic tumors. (a) Ki-67 nuclear expression was positive in $2 \%$ of tumor cell in this well-differentiated tumor. (b) CD34 identifies some capillary vessels (arrows) in this well-differentiated tumors, which was considered at low microvessel density. (c) p53 nuclear expression is high in this tumor: it is expressed by more than $80 \%$ of tumor cells. (d) HIF-1 $\alpha$ nuclear expression is strongly detected around necrosis (N) in this aggressive and mitotic, moderately differentiated tumor. (e) VEGF is diffusely and moderately expressed in a well-differentiated tumor. (f) Cytoplasmic expression of Akt is diffused in this tumor. Akt is not expressed by the tumor stroma. (g) CA9 expression is very strong and membranous in this tumor. (h) Cytoplasmic expression of $\mathrm{Bcl}-2$ is moderate, detected in more than $50 \%$ of tumor cells. (i) MDR1 staining is cytoplasmic and diffused in tumor cells. In contrast, a pancreatic duct is strongly detected with a membranous staining (arrow). (j) PTEN expression is strong and cytoplasmic in this tumor. It is not expressed in the adjacent liver (L). (k) Cytoplasmic expression of thymidylate synthase is faint in most tumor cells, whereas nuclear expression is strong in $5 \%$ of tumor cells. (I) hMLH1 is faintly detected in both the cytoplasm and the nucleus of most tumor cells.

\section{CA9}

CA9 expression was both cytoplasmic and membranous. Its score ranged from 0 to 200 (median: 57; mean: 106); $50 \%$ had high expression (Fig. 1g). There was no expression of CA9 on tumor cell nuclei. Some CA9 expression was detected on the stroma or vessels but this was not taken into account. Higher CA9 correlated with poorly differentiated tumors $(P=0.04)$ and grade $\mathrm{G}_{2} / \mathrm{G}_{3}$ tumors $(P=0.004)$.

\section{Bcl-2}

The cytoplasmic score ranged from 0 to 200 (median: 0; mean: 9); $14 \%$ were considered as high expression (Fig. 1h). There was no expression of Bcl-2 in nuclei, stroma, or vessels. There was no correlation with tumor grade or differentiation.

\section{MDR1}

The MDR1 staining was cytoplasmic, either diffuse (Fig. 1i) or punctiform, and highlighted the Golgi apparatus. The cytoplasmic score ranged from 0 to 200 (median: 100; mean: 108.5); 37\% were considered as high expression. There was no expression of MDR1 in nuclei, stroma, or vessels. There was no correlation with tumor grade or differentiation.

\section{PTEN}

The cytoplasmic and nuclear scores ranged from 0 to 200 (median: 55; mean: 71.3) and from 0 to 200 (median: 0; mean: 17.9) respectively (Fig. 1j); 50 and $27 \%$ were considered as high cytoplasmic or nuclear expression respectively. There was no expression of PTEN on stroma or vessels. PTEN cytoplasmic correlated with higher tumor grade $(P=0.02)$. PTEN nuclear did not correlate with either tumor grade or differentiation.

\section{Thymidylate synthase}

The cytoplasmic and nuclear scores ranged from 0 to 200 (median: 100; mean: 84.16) and from 0 to 200 (median: 30; mean: 56.12) respectively (Fig. 1k); 16.6 and $48 \%$ were considered as high cytoplasmic or nuclear expression respectively. There was no expression of TS in stroma or vessel. There was no correlation with tumor grade or differentiation.

\section{$h M L H 1$}

The cytoplasmic and nuclear scores ranged from 0 to 200 (median: 55; mean: 71.33) and from 0 to 200 (median: 0; mean: 17.92) respectively (Fig. 11); 50 and $28.3 \%$ were considered as high cytoplasmic or nuclear 
expression respectively. There was no expression of hMLH1 in stroma or vessel. There was no correlation with tumor grade or differentiation.

\section{Response to chemotherapy and chemoembolization}

Thirty-seven patients received streptozotocin; similarly, 19 received doxorubicin and 18 had 5-FU. Sixteen patients had the combination of etoposide/cisplatinum. Following one course of chemotherapy responders included streptozotocin (8), doxorubicin (8), 5-FU (2), and etoposide/cisplatinum (5). For the entire group, Akt was predictive of a nonresponse to treatment and PTEN to a positive response to therapy at univariate analysis $(P=0.05$ and 0.05 respectively). Mean high expression of the following predicted a lack of response to the following cytotoxics (Table 2): streptozotocin, Akt $(P=0.07)$; doxorubicin, Ki-67 $(P=0.05)$, Akt $(P=0.06)$ and CA9 $(P=0.02)$. Mean high expression of TS $(P=0.02)$ and PTEN $(P=0.017)$ was associated with a response to streptozotocin. No markers were significant for treatment with 5-FU. For well-differentiated tumors, high mean Akt expression $(P<0.03)$ was associated with a nonresponse to chemotherapy regardless of type of treatment; using multivariate analysis, only high Akt expression significantly predicted a nonresponse to therapy (OR: $0.2(0.05-0.8)$ ).

\section{Response to systemic chemotherapy}

When analysis was restricted to patients receiving systemic chemotherapy alone $(n=48)$, expression of PTEN $(P=0.04)$ and hLMH1 $(P=0.03)$ correlated with response to treatment, whereas Ki-67 $(P=0.01)$ and p53 $(P=0.06)$ expressions were associated with a lack of response or progression to therapy. High mean PTEN and hLMH1 expressions were respectively associated with a response to therapy in patients receiving streptozotocin $(P=0.008)$ and doxorubicin $(P=0.09)$. Weak expression of Akt (0.09) and CA9 $(P=0.09)$ was associated with a response to doxorubicin. Ki-67 $(P=0.006)$ and p53 $(0.06)$ expressions were associated with progressive disease to streptozotocin. Similarly, to the entire cohort, no markers were associated with a response to 5-FU, although lack of response was associated with $\mathrm{Ki}-67(P=0.004)$, MDR $(P=0.04)$, and p53 $(P=0.06)$.

\section{Survival (prognostic biomarkers)}

At the time of analysis, 31 (51.7\%) patients had died of disease (Fig. 2a). The median survival for patients with well-differentiated and poorly differentiated tumors was 110 months $(0-155)$ and 13 months $(0-112)$ respectively. Survival rates at 1,3 , and 5 years were respectively as follows: 90,70 , and $56 \%$. For the overall cohort, a number of markers were associated with overall survival (Figs 2 and 3): Ki-67, $P<0.001$; tumor grade, $P<0.001$; tumor differentiation, $P<0.001$; CA9, $P=0.004$; Akt, $P=0.01$; HIF-1, $P<0.001$; p53, $P<0.0001$; and cythMLH1, $P=0.005$. For welldifferentiated tumors $(n=47)$, Ki-67 proliferation index $(P=0.01)$ and tumor grade $(P<0.001)$ were significantly associated with survival (Fig. 3 ).

\section{Discussion}

While GEP tumors have a reputation for having an indolent nature, overall 5-year survival for welldifferentiated metastatic disease is usually $<40 \%$,
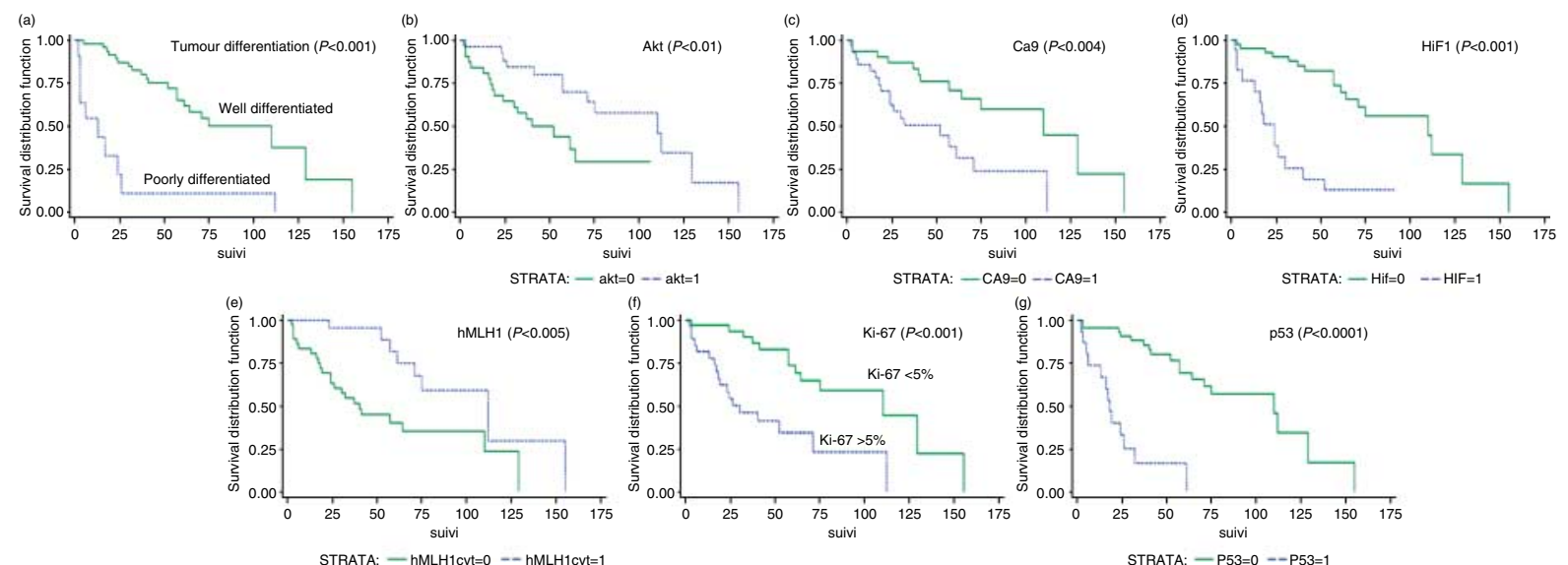

STRATA: - hMLH1cyt=0 -- hMLH1cyt=

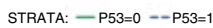

Figure 2 Survival (months) in all patients ( $n=60)$ according to (a) tumor differentiation, (b) Akt expression, (c) Ca9 expression, (d) HIF-1 expression, (e) hLMH1 expression, (f) Ki-67 expression, and (g) p53 expression. 
(a)

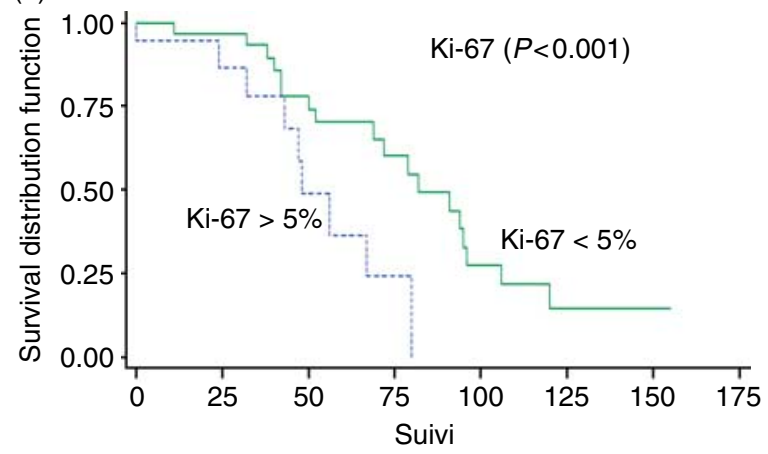

(b)

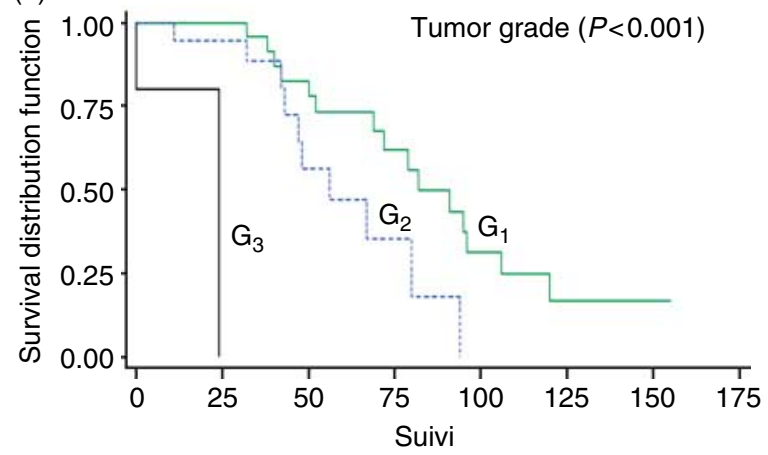

Figure 3 Survival of patients $(n=47)$ with well-differentiated tumors (months). (a) According to Ki-67 level. (b) According to tumor grade.

especially with progressive hepatic metastases (Steinmuller et al. 2008). Here, we confirm the interest of $\mathrm{Ki}-67$ as a prognostic biomarker as it was significantly associated with survival both in the entire cohort and separately in well-differentiated tumors. Survival was also predicted by the grading system proposed in the TNM/grading classification by ENETS (Rindi et al. 2006, 2007). Grading was associated with survival in a number of recent studies in pancreatic GEP (Pape et al. 2008, La Rosa et al. 2009); however, it also appears useful in a wider range of GEP and even when restricted to well-differentiated tumors. Similarly, p53 correlated with tumor differentiation, grade, and survival. Hypoxia-regulating proteins have been implicated in aggressive tumor phenotypes and poor response to therapy. Both HIF- $1 \alpha$ and CA9 significantly correlated with poorly differentiated and $\mathrm{G}_{2} / \mathrm{G}_{3}$ tumors. Recent data from our group revealed both HIF- $1 \alpha$ and CA9 as poor prognostic markers in pancreatic GEP (Couvelard et al. 2005, 2008), and here, we show that these markers are also applicable in predicting survival to GEP from intestinal origin. Interestingly, the MMR protein, hLMH1, was associated with an aggressive phenotype in our cohort of patients, and was also associated with a response to treatment with in patients receiving systemic chemotherapy $(P=0.03)$ as has been described in other solid tumors (Kitajima et al. 2003, Ide et al. 2008, Honecker et al. 2009).

Identification of markers capable of predicting responders or nonresponders to therapy could allow for a tailored approach to therapy and avoid use of potential toxic protocols in predictable nonresponders. Indeed, such an approach was recently described in pancreatic GEP where a low level O6-methylguanineDNA methyltransferase (MGMT), a key enzyme in DNA repair, was suggested to be responsible for the lack of response to temozolamide (Kulke et al. 2009). Further studies examining MGMT status are currently underway. Here, 5 of 12 markers were significantly associated with a response to cytotoxics in welldifferentiated tumors. Akt was associated with a lack of response to either streptozotocin or doxorubicin in the entire cohort. Interestingly, Akt status appears quite important, as it remained correlated with response regardless of the differentiation status. Similar to previous reports in other tumors (Dhawan et al. 2002), Akt was also found to predict survival. Chemotherapyinduced activation of Akt has been reported to confer resistance to treatment in some cellular systems (Huang $\&$ Hung 2009). However, this appears to be a complex system as recently in two NET cell lines treated with the mammalian target of rapamycin (mTOR) inhibitor rapamycin; Akt was found to be induced despite significant inhibition of cellular proliferation (Moreno et al. 2008). When analysis was restricted to systemic chemotherapy alone, lack of Akt expression remained correlated with response to doxorubicin.

Little data are available for PTEN in GEP tumors, which acts as part of the PTEN/Akt pathway, and have been implicated in cancerogenesis in many tumors (Blanco-Aparicio et al. 2007). Most carcinoids express PTEN but over half of poorly differentiated GEP demonstrate loss of PTEN (Wang et al. 2002), and altered subcellular localization of PTEN has also been reported in islet cell carcinoma (Perren et al. 2000). In this study, cytoplasmic PTEN expression was associated with a response to therapy in the entire patient cohort $(P=0.05)$, and this association was maintained when confined to patients receiving only systemic chemotherapy $(P=0.04)$. PTEN expression was specifically associated with a favorable outcome in patients receiving streptozotocin.

Although high TS has predicted poor response to therapy in a range of tumors (Pestalozzi et al. 1997, Huang et al. 2000, Harpole et al. 2001, Mizutani et al. 2001, Lurje et al. 2009), little is known of its possible role as a therapeutic marker in GEPs. Recently, in 
Table 3 Response to chemotherapy

\begin{tabular}{|c|c|c|c|c|c|}
\hline \multirow[b]{2}{*}{ Differentiation } & \multicolumn{3}{|c|}{$\begin{array}{l}\text { Well-differentiated tumors } \\
(n=47)\end{array}$} & \multirow{2}{*}{$\begin{array}{l}\text { Poorly differentiated } \\
(n=13) \\
\text { Well-differentiated } \\
(n=3) \\
\text { VP16/cisplatin } \\
(N=16)\end{array}$} & \multirow[b]{2}{*}{ Total $(n=60)$} \\
\hline & $\begin{array}{l}\text { Streptozotocin } \\
(n=37)\end{array}$ & $\begin{array}{l}\text { Doxorubicin } \\
(n=19)\end{array}$ & $\begin{array}{l}5-\mathrm{FU} \\
(n=18)\end{array}$ & & \\
\hline $\begin{array}{l}\text { Response to treatment } \\
\text { (CR or PR) }\end{array}$ & 8/37 (22\%) & 8/19 (42\%) & 2/18 (11\%) & $5 / 16(31 \%)$ & \\
\hline $\begin{array}{l}\text { Marker associated with } \\
\text { response to therapy }\end{array}$ & \multicolumn{4}{|c|}{$\boldsymbol{P}$ (high/low expression) $^{\mathrm{a}}$} & $P$ \\
\hline Ki-67 & $0.25(15 / 37)$ & $0.05(8 / 19)$ & $0.53(7 / 18)$ & $1.00(13 / 16)$ & 0.17 \\
\hline p53 & $0.64(7 / 37)$ & $0.60(4 / 19)$ & $1.00(3 / 18)$ & $1.00(9 / 16)$ & 0.75 \\
\hline Akt & $0.07(21 / 37)$ & $0.06(10 / 19)$ & $1.00(11 / 18)$ & $1.00(3 / 16)$ & 0.05 \\
\hline PTEN & $0.01(12 / 37)$ & $1.00(9 / 19)$ & $0.31(4 / 18)$ & $0.56(4 / 16)$ & 0.05 \\
\hline MDR1 & $0.14(15 / 37)$ & $1.00(6 / 19)$ & $0.18(9 / 18)$ & $0.60(5 / 16)$ & 0.49 \\
\hline VEGF & $0.71(19 / 37)$ & $0.65(11 / 19)$ & $1.00(7 / 18)$ & $0.25(5 / 16)$ & 0.35 \\
\hline CD34 & $1.00(19 / 37)$ & $1.00(9 / 19)$ & $1.00(10 / 18)$ & $0.23(4 / 16)$ & 0.23 \\
\hline HIF-1 $1 \alpha$ & $0.65(6 / 37)$ & 0.60 (4/19) & $1.00(2 / 18)$ & $0.60(11 / 16)$ & 0.86 \\
\hline CA9 & $0.14(16 / 37)$ & $0.02(9 / 19)$ & $0.49(8 / 18)$ & $0.10(11 / 16)$ & 0.33 \\
\hline $\mathrm{Bcl}-2$ & $1.00(8 / 37)$ & $1.00(0 / 19)$ & $1.00(3 / 18)$ & $1.00(5 / 16)$ & 1.00 \\
\hline TS & $0.02(8 / 37)$ & $1.00(1 / 19)$ & $1.00(5 / 18)$ & $1.00(2 / 16)$ & 0.08 \\
\hline hLMH1 & $1.00(11 / 37)$ & $1.00(8 / 19)$ & $0.49(7 / 18)$ & $0.60(3 / 16)$ & 0.76 \\
\hline
\end{tabular}

CR, complete response; PR, minor response.

aExpressed as a function of median cut-off values as follows: Ki-67: $\geq 5 \%$; p53: 0 (scores > 5 considered high); Akt: 100; PTEN: 55; MDR1: 100; VEGF: 50; CD34: 100; HIF-1 $\alpha$ : 57.5; CA9: 57; Bcl-2: 0; TS: 30; hLMH1: 55.

patients with well-differentiated GEP treated with 5-FU, high TS mRNA levels were associated with shorter time to progression and overall survival (Ceppi et al. 2008). We demonstrated that high TS was associated with poor treatment response to streptozotocin but not to 5-FU. In fact, when 5-FU was considered independently, none of the markers tested were significant (however, only 2 of 18 patient responders to 5-FU-based chemotherapy in our study may explain this result). While chemoresistance to doxorubicin has been associated with induction of HIF- $1 \alpha$-mediated angiogenesis in certain cellular systems (Mi et al. 2008), this factor was not associated with a response to therapy in this study. Nonetheless, a closely related hypoxia factor, CA9, was associated with a poor response to doxorubicin in the overall cohort $(P=0.02)$ and in patients receiving only systemic chemotherapy $(P=0.07)$. Ki-67 proliferation index was associated with a response to doxorubicin in the entire cohort of patients and along with p53 were negatively correlated with response to therapy in patients treated with systemic therapy alone. While Ki-67 has traditionally been recognized as a modest prognostic factor, recent neoadjuvant studies in breast cancer suggest that on-treatment measurement may be a more effective predictor of treatment efficacy for both endocrine treatment and chemotherapy (Dowsett \& Dunbier 2008, Miglietta et al. 2009). Using tissue arrays, we previously found a differential expression of MDR1 in metastatic versus nonmetastatic pancreatic GEP $(P=0.0003$; Couvelard et al. 2006). In this study, MDR1 was not correlated with tumor grade or differentiation, although its expression was associated with lack of response to 5-FU in those receiving systemic chemotherapy (Table 3 ).

It is important to address the potential weaknesses of this study. 1) The fact that some patients received chemoembolization with either streptozotocin or doxorubicin introduces heterogeneity; however, we attempted to adjust for this by performing an independent analysis in patients receiving only systemic chemotherapy. Indeed, embolization on its own can lead to reasonable response rates, and we cannot exclude the possibility that some of our markers may indicate an hypoxic or angiogenic phenotype in relation to embolization. However, of the factors examined related to angiogenesis (Akt, VEGF, HIF-1 $\alpha$, CD34, and CA9), only Akt and CA9 appeared important here. 2) The number of patients in the entire cohort and those receiving systemic chemotherapy alone are low 
and drawing substantial conclusions regarding reliable bio-predictors to cytotoxics should be cautioned, and further studies are required to confirm these results. The significance of markers with marginal $P$ values may become more apparent with a much larger group of patients; however, performing such studies in GEP patient populations are not easy due to their heterogeneity and relative rarity.

In conclusion, our results indicate a number of new prognostic biomarkers in GEP tumors. Additionally, we reveal for the first time that a number of biomarkers reveal potential interest in predicting response to chemotherapy in advanced or metastatic GEP, independently of their site of origin. Further studies using markers associated with a response to therapy such as CA9, Akt, PTEN, TS, and hLMH1 need to be applied and tested prospectively, preferable in a randomized setting, examining outcomes to current cytotoxic protocols.

\section{Declaration of interest}

The authors declare that there is no conflict of interest that could be perceived as prejudicing the impartiality of the research reported.

\section{Funding}

This work was sponsored by an Educational Grand from the Nelia and Amadeo Barletta Foundation.

\section{References}

Allegra CJ, Jessup JM, Somerfield MR, Hamilton SR, Hammond EH, Hayes DF, McAllister PK, Morton RF \& Schilsky RL 2009 American Society of Clinical Oncology provisional clinical opinion: testing for KRAS gene mutations in patients with metastatic colorectal carcinoma to predict response to anti-epidermal growth factor receptor monoclonal antibody therapy. Journal of Clinical Oncology 27 2091-2096. (doi:10.1200/JCO. 2009.21.9170)

Bendardaf R, Lamlum H, Ristamaki R, Syrjanen K \& Pyrhonen S 2008 Oncoprotein Bcl-2 and microsatellite instability are associated with disease-free survival and treatment response in colorectal cancer. Oncology Reports 20 999-1004. (doi:10.3892/or_00000101)

Blanco-Aparicio C, Renner O, Leal JF \& Carnero A 2007 PTEN more than the AKT pathway. Carcinogenesis 28 1379-1386. (doi:10.1093/carcin/bgm052)

Ceppi P, Volante M, Ferrero A, Righi L, Rapa I, Rosas R, Berruti A, Dogliotti L, Scagliotti GV \& Papotti M 2008 Thymidylate synthase expression in gastroenteropancreatic and pulmonary neuroendocrine tumors. Clinical Cancer Research 14 1059-1064. (doi:10.1158/10780432.CCR-07-1513)
Couvelard A, O'Toole D, Turley H, Leek R, Sauvanet A, Degott C, Ruszniewski P, Belghiti J, Harris AL, Gatter K et al. 2005 Microvascular density and hypoxia-inducible factor pathway in pancreatic endocrine tumours: negative correlation of microvascular density and VEGF expression with tumour progression. British Journal of Cancer 92 94-101. (doi:10.1038/sj.bjc.6602245)

Couvelard A, Hu J, Steers G, O'Toole D, Sauvanet A, Belghiti J, Bedossa P, Gatter K, Ruszniewski P \& Pezzella F 2006 Identification of potential therapeutic targets by gene-expression profiling in pancreatic endocrine tumors. Gastroenterology 131 1597-1610. (doi:10.1053/j.gastro.2006.09.007)

Couvelard A, Deschamps L, Rebours V, Sauvanet A, Gatter K, Pezzella F, Ruszniewski P \& Bedossa P 2008 Overexpression of the oxygen sensors PHD-1, PHD-2, PHD-3, and FIH Is associated with tumor aggressiveness in pancreatic endocrine tumors. Clinical Cancer Research 14 6634-6639. (doi:10.1158/1078-0432.CCR-07-5258)

Dhawan P, Singh AB, Ellis DL \& Richmond A 2002 Constitutive activation of $\mathrm{Akt} /$ protein kinase $\mathrm{B}$ in melanoma leads to up-regulation of nuclear factor- $\kappa \mathrm{B}$ and tumor progression. Cancer Research 62 7335-7342.

Dowsett M \& Dunbier AK 2008 Emerging biomarkers and new understanding of traditional markers in personalized therapy for breast cancer. Clinical Cancer Research 14 8019-8026. (doi:10.1158/1078-0432.CCR-08-0974)

Harpole DH Jr, Moore MB, Herndon JE II, Aloia T, D'Amico TA, Sporn T, Parr A, Linoila I \& Allegra C 2001 The prognostic value of molecular marker analysis in patients treated with trimodality therapy for esophageal cancer. Clinical Cancer Research 7 562-569.

Honecker F, Wermann H, Mayer F, Gillis AJ, Stoop H, van Gurp RJ, Oechsle K, Steyerberg E, Hartmann JT, Dinjens WN et al. 2009 Microsatellite instability, mismatch repair deficiency, and BRAF mutation in treatment-resistant germ cell tumors. Journal of Clinical Oncology 27 2129-2136. (doi:10.1200/JCO. 2008.18.8623)

Huang WC \& Hung MC 2009 Induction of akt activity by chemotherapy confers acquired resistance. Journal of the Formosan Medical Association 108 180-194. (doi:10. 1016/S0929-6646(09)60051-6)

Huang CL, Yokomise H, Kobayashi S, Fukushima M, Hitomi S \& Wada H 2000 Intratumoral expression of thymidylate synthase and dihydropyrimidine dehydrogenase in non-small cell lung cancer patients treated with 5-FU-based chemotherapy. International Journal of Oncology 17 47-54.

Ide T, Kitajima Y, Ohtaka K, Mitsuno M, Nakafusa Y \& Miyazaki K 2008 Expression of the hMLH1 gene is a possible predictor for the clinical response to 5-fluorouracil after a surgical resection in colorectal cancer. Oncology Reports 19 1571-1576.

Kitajima Y, Miyazaki K, Matsukura S, Tanaka M \& Sekiguchi M 2003 Loss of expression of DNA repair 
enzymes MGMT, hMLH1, and hMSH2 during tumor progression in gastric cancer. Gastric Cancer 6 86-95. (doi:10.1007/s10120-003-0213-z)

Kulke MH, Hornick JL, Frauenhoffer C, Hooshmand S, Ryan DP, Enzinger PC, Meyerhardt JA, Clark JW, Stuart K, Fuchs CS et al. 2009 O6-methylguanine DNA methyltransferase deficiency and response to temozolomide-based therapy in patients with neuroendocrine tumors. Clinical Cancer Research $\mathbf{1 5}$ 338-345. (doi:10.1158/1078-0432.CCR-08-1476)

La Rosa S, Klersy C, Uccella S, Dainese L, Albarello L, Sonzogni A, Doglioni C, Capella C \& Solcia E 2009 Improved histologic and clinicopathologic criteria for prognostic evaluation of pancreatic endocrine tumors. Human Pathology 40 30-40. (doi:10.1016/j.humpath.2008.06.005)

Loupakis F, Pollina L, Stasi I, Ruzzo A, Scartozzi M, Santini D, Masi G, Graziano F, Cremolini C, Rulli E et al. 2009 PTEN expression and KRAS mutations on primary tumors and metastases in the prediction of benefit from cetuximab plus irinotecan for patients with metastatic colorectal cancer. Journal of Clinical Oncology 27 2622-2629. (doi:10.1200/ JCO.2008.20.2796)

Lurje G, Manegold PC, Ning Y, Pohl A, Zhang W \& Lenz HJ 2009 Thymidylate synthase gene variations: predictive and prognostic markers. Molecular Cancer Therapeutics 8 1000-1007. (doi:10.1158/1535-7163.MCT-08-0219)

Mi J, Zhang X, Rabbani ZN, Liu Y, Reddy SK, Su Z, Salahuddin FK, Viles K, Giangrande PH, Dewhirst MW et al. 2008 RNA aptamer-targeted inhibition of NF- $\kappa$ B suppresses nonsmall cell lung cancer resistance to doxorubicin. Molecular Therapy 16 66-73. (doi:10.1038/sj.mt.6300320)

Miglietta L, Vanella P, Canobbio L, Parodi MA, Guglielmini P \& Boccardo F 2009 Clinical and pathological response to primary chemotherapy in patients with locally advanced breast cancer grouped according to hormonal receptors, her2 status, grading and ki-67 proliferation index. Anticancer Research 29 1621-1625.

Mizutani Y, Wada H, Ogawa O, Yoshida O, Fukushima M, Nonomura N \& Miki T 2001 Prognostic significance of thymidylate synthase activity in bladder carcinoma. Cancer 92 510-518. (doi:10.1002/1097-0142(20010801) 92:3<510::AID-CNCR1349>3.0.CO;2-0)

Modok S, Mellor HR \& Callaghan R 2006 Modulation of multidrug resistance efflux pump activity to overcome chemoresistance in cancer. Current Opinion in Pharmacology 6 350-354. (doi:10.1016/j.coph.2006.01.009)

Moreno A, Akcakanat A, Munsell MF, Soni A, Yao JC \& Meric-Bernstam F 2008 Antitumor activity of rapamycin and octreotide as single agents or in combination in neuroendocrine tumors. Endocrine-Related Cancer 15 257-266. (doi:10.1677/ERC-07-0202)

O'Toole D 2006 Current trend: endocrine tumors of the stomach, small bowel, colon and rectum. Gastroenterologie Clinique et Biologique 30 276-291. (doi:10.1016/ S0399-8320(06)73165-7)
O’Toole D, Hentic O, Corcos O \& Ruszniewski P 2004 Chemotherapy for gastro-enteropancreatic endocrine tumours. Neuroendocrinology 80 (Supplement 1) 79-84. (doi:10.1159/000080747)

Pape UF, Jann H, Muller-Nordhorn J, Bockelbrink A, Berndt U, Willich SN, Koch M, Rocken C, Rindi G \& Wiedenmann B 2008 Prognostic relevance of a novel TNM classification system for upper gastroenteropancreatic neuroendocrine tumors. Cancer 113 256-265. (doi:10.1002/cncr.23549)

Perren A, Komminoth P, Saremaslani P, Matter C, Feurer S, Lees JA, Heitz PU \& Eng C 2000 Mutation and expression analyses reveal differential subcellular compartmentalization of PTEN in endocrine pancreatic tumors compared to normal islet cells. American Journal of Pathology 157 1097-1103.

Pestalozzi BC, Peterson HF, Gelber RD, Goldhirsch A, Gusterson BA, Trihia H, Lindtner J, Cortes-Funes H, Simmoncini E, Byrne MJ et al. 1997 Prognostic importance of thymidylate synthase expression in early breast cancer. Journal of Clinical Oncology 15 1923-1931.

Rindi G, Kloppel G, Alhman H, Caplin M, Couvelard A, de Herder WW, Erikssson B, Falchetti A, Falconi M, Komminoth P et al. 2006 TNM staging of foregut (neuro)endocrine tumors: a consensus proposal including a grading system. Virchows Archiv 449 395-401. (doi:10. 1007/s00428-006-0250-1)

Rindi G, Kloppel G, Couvelard A, Komminoth P, Korner M, Lopes JM, McNicol AM, Nilsson O, Perren A, Scarpa A et al. 2007 TNM staging of midgut and hindgut (neuro) endocrine tumors: a consensus proposal including a grading system. Virchows Archiv 451 757-762. (doi:10. 1007/s00428-007-0452-1)

Steinmuller T, Kianmanesh R, Falconi M, Scarpa A, Taal B, Kwekkeboom DJ, Lopes JM, Perren A, Nikou G, Yao J et al. 2008 Consensus guidelines for the management of patients with liver metastases from digestive (neuro)endocrine tumors: foregut, midgut, hindgut, and unknown primary. Neuroendocrinology 87 47-62. (doi:10.1159/ 000111037)

Tan EY, Yan M, Campo L, Han C, Takano E, Turley H, Candiloro I, Pezzella F, Gatter KC, Millar EK et al. 2009 The key hypoxia regulated gene CAIX is upregulated in basal-like breast tumours and is associated with resistance to chemotherapy. British Journal of Cancer 100 405-411. (doi:10.1038/sj.bjc. 6604844)

Wang L, Ignat A \& Axiotis CA 2002 Differential expression of the PTEN tumor suppressor protein in fetal and adult neuroendocrine tissues and tumors: progressive loss of PTEN expression in poorly differentiated neuroendocrine neoplasms. Applied Immunohistochemistry and Molecular Morphology 10 139-146. (doi:10.1097/00022744200206000-00008) 